Journal of Applied Pharmaceutical Science Vol. 7 (06), pp. 229-237, June, 2017

Available online at http://www.japsonline.com

DOI: $10.7324 / \mathrm{JAPS} .2017 .70634$

ISSN 2231-3354 (cc)) EY-NC-SA

\title{
An Overview of Naturally Synthesized Metallic Nanoparticles
}

\author{
Mariappan Vignesh, Puthamohan Vinayaga Moorthi* \\ Department of Human Genetics and Molecular Biology, School of Life sciences, Bharathiar University, Coimbatore- 641 046, India.
}

\begin{tabular}{|c|c|}
\hline ARTICLE INFO & ABSTRACT \\
\hline $\begin{array}{l}\text { Article history: } \\
\text { Received on: } 16 / 12 / 2016 \\
\text { Accepted on: } 27 / 02 / 2017 \\
\text { Available online: } 30 / 06 / 2017\end{array}$ & $\begin{array}{l}\text { Nanotechnology is a powerful technology offers wide spectrum of devices, drugs etc for the welfare of human } \\
\text { and other living organisms. Nanoparticles are the tiny molecule in the size of } 1-100 \mathrm{~nm} \text { acting as potential } \\
\text { antimicrobial, anticancer, Anti-Larvicidal and Antiparasitic Agents. Among the nanoparticles deployed, silver } \\
\text { and gold nanoparticles have been greatly used against multidrug resistant microbes like Staphylococcus aureus, }\end{array}$ \\
\hline $\begin{array}{l}\text { Key words: } \\
\text { Nanoparticles, Antimicrobial, } \\
\text { Anticancer, Larvicidal } \\
\text { activity, MCF-7. }\end{array}$ & $\begin{array}{l}\text { vector mosquitoes like Aedes aegypti, Anopheles stephensi and Culex quinquifasciatus as well as wide range of } \\
\text { cancer Cell lines like MCF-7, Hep G2, etc. In the recent years, identification of dynamic nanoparticles, } \\
\text { possessing antimicrobial, anti-larvicidal and anticancer activity, becomes important for defending the existing } \\
\text { multitude of diseases spreading to human beings. In this context, this review was prepared to measure the } \\
\text { dynamics of bioactive nanoparticles extremely used against pathogens, cell lines and vectors. }\end{array}$ \\
\hline
\end{tabular}

\section{INTRODUCTION}

The mosquito vectors are responsible transfer of deadly diseases such as malaria, chickungunia, filariasis and dengue. One of the major economic and health issues in several countries are the vector borne diseases caused by mosquito. The mosquito vector for human diseases like yellow fever and dengue is Aedes agypti mosquito (Suganya et al., 2014). Similarly, mosquitoborne viral disease called dengue was found mostly in tropic and sub-tropic region around the world. Estimation by World Health Organization (WHO) states that there are about 50-100 million dengue infection are prevalent worldwide (Suresh et al., 2015). A vector of Wuchereria species called Culex quinquefasciatus was responsible for causing lymphatic Filariasis which are wide spread in the tropical region with 120 million people infected and 40 million people are under clinical manifestation (Veerakumar et al., 2013). Two million malarial cases were reported

\footnotetext{
* Corresponding Author

Dr. P. Vinayaga Moorthi, Assistant Professor, Department of Human Genetics and Molecular Biology, School of Life Sciences, Bharathiar University, Coimbatore - 641 046, Tamil Nadu, INDIA.

Email: vinayputhu@gmail.com; Mobile:+91-99948-09189
}

annually in India (Gnanadesigan et al., 2011). Due to the development of insecticides resistance, biological magnification, serious effect on environmental quality and destruction of nontarget organism, the vector controlling methods involving chemical insecticides are becoming less effective (Suganya et al., 2014). The use of artificial insecticides in order to control mosquito-borne vector especially Aedes leads to high cost and harmful non-target effect (Suresh et al., 2015). Cancer, which is grouped as the uncontrolled development of cells, is one of the serious causes of death throughout the world. In the last few years, Increase of cancer patient have been observed (Abbas et al., 2015). The bites of Aedes agypti can transmit hamster reticulum cell sarcoma by transfer of tumor cell. They also plays role in changing the human metabolic pathway which lead to viral infection or/and oncogenesis (Benelli et al., 2016). There are several ways in the treatment of cancer by chemotherapy while there are multiple barriers that recently prevent the clinical advantages. Currently, the field of nanotechnology plays a major role in the cancer treatment through drug delivery method because of the good pharmacokinetic activity, better drug solubility, increased the halflife period of drugs. In addition to that they also have less toxic phytochemicals with target-specific nanoparticles which provide a new way of treating cancer (Joseph et al., 2015). 
Benelli, 2016 stated that nanodrugs with multipotency against mosquito-borne diseases and cancers are required. In particular, a focus on effectiveness and non-target effects of metal nanoparticles synthesized using natural products as reducing agents may support the development of novel antiplasmodial, mosquitocidal, and anticancer tools. In this vision we have prepared this review. This review mainly focuses on the pharmacokinetics activity of several nanoparticles such as antiparasitic, anticancer, antimicrobial, antioxidant and antiinflammation.

\section{AGNPS (AgNps)}

\section{Antiparasitic Study}

The AgNps (AgNps) synthesised using leaf extract of Rhizophora mucronata have excellent larvicidal activity $\left(\mathrm{LC}_{50}\right.$ and $\mathrm{LC}_{90}$ ) against the larvae of Aedes aegypti and Culex quinquefasciatus with respect to $0.585 \mathrm{mg} / \mathrm{L}$ and $2.615 \mathrm{mg} / \mathrm{L}$ and 0.891 and $6.291 \mathrm{mg} / \mathrm{mL}$ (Gnanadesigan et al., 2011). The AgNps synthesised through aqueous extract of stem of Cissus quadrangularis have excellent adulticidal activity ( $\mathrm{LC}_{50}$ and $\mathrm{LC}_{90}$ ) against Hippobosca maculata with respect to 37.08, 40.35 and $6.30 \mathrm{mg} / \mathrm{L}$ as well as $175.46,192.17$ and $18.14 \mathrm{mg} / \mathrm{L}$. It also revealed larvicidal activity against Rhipicephalus (Boophilus) microplus $\left(\mathrm{LC}_{50}\right.$ values of 50.00, 21.72 and $7.61 \mathrm{mg} / \mathrm{L} ; \mathrm{LC}_{90}$ values of 205.12, 82.99 and $22.68 \mathrm{mg} /$ ) (Santhoshkumar et al., 2012).

The mesocarp layer extract Cocos nucifera coir engineered AgNps have potential larvicidal activity against Anopheles stephensi and C. quinquefasciatus with an $\mathrm{LC}_{50}$ value of 87.24 and 49.89 as well as $\mathrm{LC}_{90}$ values of 230.90 and $84.85 \mathrm{mg} / \mathrm{L}$ respectively (Roopan et al., 2012). AgNps from bark aqueous extract of Ficus racemosa have shown excellent larvicidal activity against $C$. quinquefasciatus $\left(\mathrm{LC}_{50}=67.72\right.$ and $\left.63.70 \mathrm{mg} / \mathrm{L}\right)$ and $C$. gelidus $\left(\mathrm{LC}_{50}=12.00\right.$ and $\left.11.21 \mathrm{mg} / \mathrm{L}\right)$ (Velayutham et al., 2012).

Similarly, AgNps from aqueous aerial extract of Ammannia baccifera represented dynamic activity against mosquito larvae namely $A$. subpictus $\left(\mathrm{LC}_{50}=257.16\right)$ and $C$. quinquefasciatus $\left(\mathrm{LC}_{50}=210.88 \mathrm{ppm}\right)$. also have shown toxic $\left(\mathrm{LC}_{50}\right)$ effect against A. subpictus and $C$. quinquefasciatus with respect to 29.54 and 22.32ppm respectively (Suman et al., 2013). Similarly AgNps form Delphinium denudatum have excellent anti-mosquito activity against second instar larvae of A. Aegypti ( $\mathrm{LC}_{50}$ : 96ppm (24hrs) and 9.6ppm (48hrs)) (Suresh et al., 2014).

AgNps engineered from extracts of leaf and fruit of Couroupita guianensis and revealed broader larvicidal activity against A. aegypti (Leaf: $\mathrm{LC}_{50}-44.55 \mathrm{ppm}$ and $\mathrm{LC}_{90}-318.39$ ppm) and (Fruit: $\mathrm{LC}_{50}-49.96 \mathrm{ppm}$ and $\mathrm{LC}_{90}-568.84 \mathrm{ppm}$ ) when compared to methanol extract (Leaf: $\mathrm{LC}_{50}-85.75 \mathrm{ppm}$ and $\mathrm{LC}_{90}-$ 598.63 ppm and (fruit; LC50 - 67.78 ppm and LC90 - 714.45 ppm). However colloid synthesis of AgNPs using aqueous extract of the $C$. quianensis by decoction method showed extensive mortiality rate against $A$. aegypti (Leaf: $\mathrm{LC}_{50}-2.1 \mathrm{ppm}$ and $\mathrm{LC}_{90}-$ $5.59 \mathrm{ppm}$ ) and (Fruit: $\mathrm{LC}_{50}-2.09 \mathrm{ppm}$ and $\mathrm{LC}_{90}-5.7 \mathrm{ppm}$ )
(Vimala et al., 2014). AgNps from leaf extract of Feronia elephantum (Rutaceae) have revealed potential antiparasitic activity $\left(\mathrm{LD}_{50}\right.$ and $\left.\mathrm{LD}_{90}\right)$ against adult of $A$. Stephensi: 18.041 and $32.575 \mu \mathrm{gmL}^{-1}, \quad$ A. Aegypti: 20.39 and $37.53 \mu \mathrm{gmL}^{-1}, \quad C$. quinquefasciatus: 21.79 and $\left.39.59 \mu \mathrm{gmL}^{-1}\right)($ Veerakumar et al., 2014).

Leaf extract of Leucas aspera synthesized AgNps portrayed potential larvicidal activity against fourth instar larvae of A. aegypti with respect to $\left(\mathrm{LC}_{50}\right.$ and $\mathrm{LC}_{90}$ values of 8.56 and $31.30 \mathrm{mg} / \mathrm{l}$ ) (Suganya et al., 2014). Aloe vera originated AgNps showed considerable toxicity against I, II, III, IV and pupae of $A$. stephensi with respect to 48.79, 59.09, 70.88, 83.58 and 152.55ppm (Dinesh et al., 2015).

Leaf extract of Annona muricata emerged AgNps exemplified excellent larvicidal activity against $A$. aegypti $\left(\mathrm{LC}_{50}\right.$ and $\mathrm{LC}_{90}$ values: 12.58 and $\left.26.46 \mu \mathrm{g} \mathrm{mL}^{-1}\right)$, A. stephensi $(15.28$ and $31.91 \mathrm{gmL}^{-1}$ ) and $C$. quinquefasciatus (18.77 and $35.72 \mu \mathrm{g}$ $\mathrm{mL}^{-1}$ ) (Santhosh et al., 2015). Larvicidal activity of Euphorbia hirta leaf extract generated AgNps found highly active against the first and fourth instar larvae and pupae of Anopheles stephensi $\left(\mathrm{LC}_{50}: 10.14,16.82,21.51\right.$, and 27.89 ppm; $\mathrm{LC}_{90}$ : 31.98, 50.38, 60.09, and $69.94 \mathrm{ppm}$ ) (Priyadarshini et al., 2012). Acetone leaf extract of Morinda tinctoria synthesized AgNps have greater larvicial activity against third instar larvae of C. quinquefasciatus ( $\mathrm{LC}_{50}$ : 8.088 and $1.442 \mathrm{ppm}$ ) (Kumar et al., 2014). The synthesised AgNps from Chomelia asiatica (Rubiaceae) has potential larvicidal activity against the mosquito vectors of $A$. stephensi, $A$. aegypti, and $C$. quinquefasciatus (Diptera: Culicidae) with the following $\mathrm{LC}_{50}$ and $\mathrm{LC}_{90}$ values. $A$. stephensi had 17.95 and $33.03 \mu \mathrm{g} / \mathrm{mL}$ while $A$. aegypti and $C$. quinquefasciatus had 19.32 and $34.87 \mu \mathrm{g} / \mathrm{mL}$ and 20.92 and 37.41 $\mu \mathrm{g} / \mathrm{mL}$ respectively (Muthukumaran et al., 2014).

The synthesised AgNps from Feronia elephantum (Rutaceae) has effective larvicidal activity against the $C$. quinquefasciatus, A. stephensi, and A. aegypti with $\mathrm{LC}_{50}$ and $\mathrm{LC}_{90}$ values of 11.56 and $20.56 \mu \mathrm{g} \mathrm{mL}^{-1}, 13.13$ and $23.12 \mu \mathrm{g} \mathrm{mL}^{-1}$, and 14.19 and $24.30 \mathrm{~g} \mathrm{~mL}^{-1}$ respectively (Veerakumar et al., 2014). AgNps from leaf extract of Gmelina asiatica had potent larvicidal activity against larvae of $A$. stephensi ( $\mathrm{LC}_{50}: 22.44$ and $\mathrm{LC}_{90}: 40.65$ $\mu \mathrm{g} / \mathrm{mL})$, Aedes aegypti $\left(\mathrm{LC}_{50}: 25.77\right.$ and $\left.\mathrm{LC}_{90}=45.98 \mu \mathrm{g} / \mathrm{mL}\right)$ and C. quinquefasciatus $\left(\mathrm{LC}_{50}: 27.83\right.$ and $\left.\mathrm{LC}_{90}=48.92 \mu \mathrm{g} / \mathrm{mL}\right)$ (Muthukumaran et al., 2015). The AgNps synthesised using aqueous fruit extract of putranjiva, Drypetes roxburghii (Wall.) has an effective larvicidal activity against $C$. quinquefasciatus ( $\mathrm{LC}_{50}$ value: $0.8632,1.1619$ and $1.2814 \mathrm{ppm}$ with respect to second, third and fourth instar larvae while A. stephensi had $\mathrm{LC}_{50}$ values of $0.7329,0.8397$ and 0.9848 ppm (Haldar et al., 2013). The AgNps synthesised from the crude methanol and aqueous extraction of Nelumbo nucifera Gaertn. (Nymphaeaceae) have excellent larvicidal activity against the fourth instar larvae of $A$. subpictus $\left(\mathrm{LC}_{50}=8.89,11.82\right.$, and $0.69 \mathrm{ppm} ; \mathrm{LC}_{90}=28.65,36.06$, and $2.15 \mathrm{ppm})$ and $C$. quinquefasciatus $\left(\mathrm{LC}_{50}=9.51,13.65\right.$, and $1.10 \mathrm{ppm} ; \mathrm{LC}_{90}=28.13,35.83$, and 3.59ppm) (Santhoshkumar et al., 2010). 
Table 1: Larvicidal activity of AgNps synthesized from different sources.

\begin{tabular}{|c|c|c|c|c|c|}
\hline \multirow{2}{*}{$\begin{array}{c}\text { BIOLOGICAL SOURCE/ } \\
\text { METHOD }\end{array}$} & \multirow{2}{*}{$\begin{array}{l}\text { SIZE } \\
(\mathbf{N M})\end{array}$} & \multirow{2}{*}{$\begin{array}{l}\text { NAME OF MOSQUITO } \\
\text { SPECIES }\end{array}$} & \multicolumn{2}{|c|}{ LETHAL CONCENTRATION (LC) (MG/L) / PPM } & \multirow{2}{*}{ REFERENCES } \\
\hline & & & $\mathbf{L C}_{50}$ & $\mathrm{LC}_{90}$ & \\
\hline \multirow{2}{*}{$\begin{array}{l}\text { Leaf extract of Rhizophora } \\
\text { mucronata }\end{array}$} & \multirow{2}{*}{$60-95$} & Aedes aegypti & 0.585 & 2.615 & \multirow{2}{*}{$\begin{array}{l}\text { Gnanadesigan et al., } \\
2011\end{array}$} \\
\hline & & Culex quinquefasciatus & 0.891 & 6.291 & \\
\hline \multirow[b]{2}{*}{$\begin{array}{l}\text { Stem Aqueous extract of Cissus } \\
\text { quadrangularis }\end{array}$} & \multirow[b]{2}{*}{42.46} & Hippobosca maculata & $37.08,40.35,6.30$ & $175.46,192.17,18.14$ & \multirow[b]{2}{*}{$\begin{array}{l}\text { Santhoshkumar } \text { et al., } \\
2012\end{array}$} \\
\hline & & $\begin{array}{c}\text { Rhipicephalus } \\
\text { (Boophilus) microplus }\end{array}$ & $50.00,21.72,7.61$ & $205.12,82.99,22.68$ & \\
\hline \multirow{2}{*}{$\begin{array}{c}\text { Mesocarplayer extract of Cocos } \\
\text { nucifera }\end{array}$} & \multirow{2}{*}{$23 \pm 2$} & Anopheles stephensi & 87.24 & $230.90 \pm 17.10$ & \multirow{2}{*}{ Roopan et al., 2012} \\
\hline & & Culex quinquefasciatus & 49.89 & $84.85 \pm 6.50$ & \\
\hline \multirow{2}{*}{$\begin{array}{c}\text { Bark aqueous extract of Ficus } \\
\text { racemosa }\end{array}$} & \multirow{2}{*}{250.6} & Culex quinquefasciatus & $67.72,63.70$ & -- & \multirow{2}{*}{$\begin{array}{l}\text { Velayutham et al., } \\
2012\end{array}$} \\
\hline & & Culex gelidus & $12.00,11.21$ & -- & \\
\hline \multirow{2}{*}{$\begin{array}{c}\text { Aqueous aerial extract of } \\
\text { Ammannia baccifera }\end{array}$} & \multirow{2}{*}{$10-30$} & Anopheles subpictus & 257.16 & -- & \multirow{2}{*}{ Suman et al., 2013} \\
\hline & & Culex quinquefasciatus & 210.88 & -- & \\
\hline $\begin{array}{l}\text { Aqueous root extract of } \\
\text { Delphinium denudatum }\end{array}$ & 85 & Aedes aegypti & $96,9.6$ & -- & Suresh et al., 2014 \\
\hline $\begin{array}{l}\text { Ethyl acetate extract of leaf and } \\
\text { fruit of Couroupita guianensis } \\
\text { Aubl. }\end{array}$ & $\begin{array}{l}\text { Leaf: } \\
\text { 10-45 } \\
\text { Fruit: } \\
5-15\end{array}$ & Aedes aegypti & $\begin{array}{l}\text { Leaf: } 44.55 \\
\text { Fruit: } 49.96\end{array}$ & $\begin{array}{l}\text { Leaf: } 318.39 \\
\text { Fruit: } 568.84\end{array}$ & Vimala et al., 2014 \\
\hline $\begin{array}{c}\text { Methanol extract of Couroupita } \\
\text { guianensis Aubl. }\end{array}$ & & Aedes aegypti & $\begin{array}{l}\text { Leaf: } 85.75 \\
\text { Fruit: } 67.78\end{array}$ & $\begin{array}{l}\text { Leaf: } 598.63 \\
\text { Fruit: } 714.45\end{array}$ & Vimala et al., 2014 \\
\hline $\begin{array}{c}\text { Colloid synthesis of aqueous } \\
\text { extract of Couroupita guianensis } \\
\text { Aubl }\end{array}$ & & Aedes aegypti & $\begin{array}{l}\text { Leaf: } 2.1 \\
\text { Fruit: } 5.59\end{array}$ & $\begin{array}{l}\text { Leaf: } 2.09 \\
\text { Fruit: } 5.7\end{array}$ & Vimala et al., 2014 \\
\hline Leafs of Melia azedarach using & & Aedes aegypti & 4.27 & 12.61 & \\
\hline $\begin{array}{c}\text { 2,7.bis[2-[diethylamino]-ethoxy] } \\
\text { fluorence }\end{array}$ & $3-31$ & Culex quinquefasciatus & 3.43 & 10.29 & Ramanibai et al., 2014 \\
\hline & & Anopheles stephensi & 18.041 & 32.575 & \\
\hline Leaf extract of Feronia & $18-45$ & Aedes aegypti & 20.399 & 37.534 & Veerakumar et al., \\
\hline & & Culex quinquefasciatus & 21.798 & 39.596 & \\
\hline Leaf extract of Leucas aspera & $25-80$ & Aedes aegypti & $\begin{array}{l}8.56,10.03,14.46 \\
13.45,17.41,27.49 \\
\end{array}$ & $\begin{array}{l}\begin{array}{l}21.5685,93.0392,39.6485, \\
42.2029,31.3009,53.2576\end{array} \\
\end{array}$ & Suganya et al., 2014 \\
\hline Leaf extract of Aloe vera & $35-55$ & Anopheles stephensi & $\begin{array}{c}48.79,59.09,70.88, \\
\quad 83.58,152.55\end{array}$ & -- & Dinesh et al., 2015 \\
\hline & & Aedes aegypti & 12.58 & 26.46 & \\
\hline Leaf extract of Annona muricata & $20-53$ & Anopheles stephensi & 15.28 & 31.91 & Santhosh et al., 2015 \\
\hline & & Culex quinquefasciatus & 18.77 & 35.72 & \\
\hline Leaf extract of Euphorbia hirta & $30-60$ & Anopheles stephensi & $\begin{array}{c}10.14,16.82,21.51, \\
27.89\end{array}$ & $31.98,50.38,60.09,69.94$ & $\begin{array}{l}\text { Priyadarshini et al., } \\
2012\end{array}$ \\
\hline $\begin{array}{l}\text { Aqueous leaf extract of Morinda } \\
\text { tinctoria using acetone }\end{array}$ & $60-95$ & Culex quinquefasciatus & $8.088,1.442$ & -- & Kumar et al., 2014 \\
\hline & & Anopheles stephensi & 17.95 & 33.03 & \\
\hline $\begin{array}{l}\text { Aqueous leat extract of } \\
\text { Chomelia asiatica (Rubiaceae) }\end{array}$ & & Aedes aegypti & 19.32 & 34.87 & $\begin{array}{l}\text { Muthukumaran et al., } \\
2014\end{array}$ \\
\hline & & Culex quinquefasciatus & 20.92 & 37.41 & \\
\hline & & Anopheles stephensi & 11.56 & 20.56 & \\
\hline elephantum (Rutaceae) & $20-60$ & Aedes aegypti & 13.13 & 23.12 & Veerakumar et al., \\
\hline & & Culex quinquefasciatus & 14.19 & 24.30 & \\
\hline & & Anopheles stephensi & 22.44 & 40.65 & \\
\hline Leaf extract of Gmelina asiatica & 32 & Aedes aegypti & 25.77 & 45.98 & Muthukumaran et al., \\
\hline & & Culex quinquefasciatus & 27.83 & 48.92 & \\
\hline Aqueous fruit extract of & & Culex quinquefasciatus & $0.86,1.16,1.28$ & -- & \\
\hline Drypetes roxburghii & 26.6 & Anopheles stephensi & $0.73,0.83,0.98$ & -- & Haldar et al., 2013 \\
\hline Crude methanol and aqueous & 45 & Anopheles subpictus & $8.89,11.82,0.69$ & $28.65,36.06,2.15$ & Santhoshkumar et al., \\
\hline extraction of Nelumbo nucifera & 45 & Culex quinquefasciatus & $9.51,13.65,1.10$ & $28.13,35.83,3.59$ & 2010 \\
\hline Aqueous leaf extract of Mukia & & Aedes aegypti & 0.211 & 0.703 & \\
\hline maderaspatana & 64 & Culex quinquefasciatus & 0.094 & 0.482 & Chitra et al., 2015 \\
\hline & & Aedes aegypti & $\begin{array}{c}13.34,17.19,22.03 \\
27.57,34.84\end{array}$ & $\begin{array}{c}36.98,47.67,55.95 \\
67.36,77.72\end{array}$ & \\
\hline Murraya koenigil leat extract & $25-35$ & Anopheles stephensi & $\begin{array}{c}10.82,14.67,19.13 \\
24.35,32.09\end{array}$ & $\begin{array}{c}32.38,42.52,53.65 \\
63.51,75.26\end{array}$ & Suganya et al., 2012 \\
\hline $\begin{array}{c}\text { Leaf aqueous extract of Nerium } \\
\text { oleander }\end{array}$ & $20-35$ & Anopheles stephensi & $\begin{array}{c}20.60,24.90,28.22, \\
33.99 \\
\end{array}$ & $41.62,50.33,57.78,68.41$ & Roni et al., 2012 \\
\hline
\end{tabular}


AgNps synthesised using aqueous leaf extract of Mukia maderaspatana (Cucurbitaceae) served as a potent larvicidal agent against $A$. aegypti and $C$. quinquefasciatus in which $\mathrm{LC}_{50}$ and $\mathrm{LC}_{90}$ of $A$. aegypti had 0.211 and $0.703 \mathrm{ppm}$ while $C$. quinquefasciatus had 0.094; 0.482ppm (Chitra et al., 2015). The AgNps synthesised from Murraya koenigii leaf extract have excellent larvicdical activity against $A$. stephensi ( $\mathrm{LC}_{50}$ values: 10.82, 14.67, 19.13, 24.35, 32.09ppm and $\mathrm{LC}_{90}$ values of 32.38, 42.52, 53.65, 63.51, $75.26 \mathrm{ppm})$ while $A$. aegypti $\left(\mathrm{LC}_{50}\right.$ values of $13.34,17.19,22.03$, 27.57, 34.84ppm and $\mathrm{LC}_{90}$ values of 36.98, 47.67, 55.95, 67.36, 77.72ppm (Suganya et al., 2012). AgNps synthesised from leaf aqueous extract of Nerium oleander have effective larvicidal activity against first to fourth instar larvae and pupae of $A$. stephensi with following values: $\mathrm{LC}_{50}$ and $\mathrm{LC}_{90}$ of instar larvae were 20.60, 24.90, 28.22, and $33.99 \mathrm{ppm}$ and 41.62, 50.33, 57.78, and $68.41 \mathrm{ppm}$ while for pupae it was 39.55 and $79.10 \mathrm{ppm}$ (Roni et al., 2012). The larvicidal activity of AgNps synthesised from different source is tabulated in table 1 .

\section{Anticancer Study}

The anticancer potential of AgNps synthesised from Acorus calamus was evaluated against epidermoid carcinoma cell (A431) (Nayak et al., 2015). The AgNps synthesised from plant polyphenol called caffeic acid through facile mediated process had shown effective anticancer activity against the human hepatoma (HepG2) cells by reducing the tumour cell viability through activating apoptosis (Guo et al., 2015). The AgNps synthesised from Andrographis echioides (leaf) through aqueous extract process have been reported with potential anticancer activity against the human breast adenocarcinoma cancer cell line (MCF-7) with an inhibitory concentration $\left(\mathrm{IC}_{50}\right)$ of $31.5 \mu \mathrm{g} / \mathrm{mL}$ at $24 \mathrm{~h}$ incubation by suppressing its growth (inhibiting the proliferation of tumour cells) (Elangovan et al., 2015). The anticancer activity of AgNps synthesized from different plant is listed in table 2.

The AgNps synthesised from ethanol extract of rose (Rosa Indica) petals have exhibited effective anticancer activity against human colon cancer cells (HCT 15 cells) by down regulating the Bcl-2 and Bax level by which upregulates activation of caspase 3 and 9 that finally induce apoptosis (Ramar et al., 2015).

The AgNps synthesised from Mollick et al. (2015) have revealed excellent anticancer activity against the Jurkat cell (human T-cell lymphoma) by reducing the viability at the rate of $52.6 \%, 85.4 \%$, and $91.6 \%$ with respect to the concentration of 10 , 25, and $50 \mu \mathrm{g} / \mathrm{ml}$ of AgNPs. Citrate coated AgNps (10nm in size) showed overall DNA damages and cytotoxic affect against human lung cells (BEAS-2B) after $24 \mathrm{~h}$ by the comet assay (Gliga et al., 2014).

\section{Antimicrobial Study}

The AgNPs synthesised from $C$. maxima (petals), $M$. oleifera (leaves) and A. calamus (rhizome) have exhibited strong antimicrobial activity against Bacillus subtilis (gram positive) and E. coli, P. aeruginosa and Vibrio cholerae (gram negative) (Nayak et al., 2015).

The AgNps synthesised from Andrographis echioides (leaf) through aqueous extract process also had excellent antibacterial activity against $E$. coli $(28 \mathrm{~mm})$ and $S$. aureus $(23 \mathrm{~mm})$ and moderate activity against Salmonella typhi (18mm), Micrococcus luteus $(15 \mathrm{~mm})$ and Pseudomonas aeruginosa (13mm) (Elangovan et al., 2015). The AgNps synthesised from rose (Rosa Indica) petals using ethanolic extraction have a potential antibacterial activities against $E$. coli (MTCC-40) and $K$. pneumonia (MTCC-740) (gram negative) than $S$. mutans (MTCC-896) and Enterococcus faecalis (MTCC-439) (Ramar et al., 2015).

The antimicrobial activities of AgNps synthesized from different plant were represented in table 3. The AgNps synthesised from Abelmoschus esculentus (L.) pulp extract have potential antibacterial activity against B. subtilis (MTCC 736), Bacillus cereus (MTCC 306), P. aeruginosa (MTCC 8158), Micrococcus luteus (MTCC 1538) and E. coli (MTCC 68) with zone of inhibition (ZoI) of 33, 28, 26, 40 and 19mm respectively (Mollick et al., 2015). Suresh et al. (2014) generated AgNps from root of Delphinium denudatum (DdAgNPs) and obtained ZoI of 10 and $8 \mathrm{~mm}$ against B. cereus (NCIM 2106) and E. coli (ATCC 8739) respectively which was comparatively lower than the activity rendered by Mollick et al. (2015).

The minimal inhibitory concentration (MIC) and minimal bactericidal concentration (MBC) of citrate stabilized AgNps synthesised using sodium citrate and found $60,160 \mathrm{mg} / \mathrm{mL}$ and 80 , $160 \mathrm{mg} / \mathrm{mL}$ against $S$. aureus and B. Megaterium respetively. Similarly, Borah et al. (2013) reported that the MIC and MBC values of AgNps synthesised from Ocimum sanctum was 40, $120 \mathrm{mg} / \mathrm{mL}$ and $80,140 \mathrm{mg} / \mathrm{mL}$ against $S$. aureus and $B$. megaterium respectively.

The AgNps from leaf extract of $O$. tenuiflorum exhibited potential antibacterial activity against E. Coli (gram negative) and Corynebacterium (gram positive) with ZoI of 10, 15, 20mm and 14, 16,20mm respectively while it was 19, 24, 26mm against B. substilus (spore forming) (Patil et al., 2012). AgNps pellets synthesised from seeds of Elaeocarpus ganitrus (Rudraksha) and Foliage of Prosopis spicigera (Shami) exhibited excellent antibacterial activity against $P$. aeruginosa and $S$. aureus with 25 and $21 \mathrm{~mm}$ (Dwivedi et al., 2013).

\section{Anti-inflammation Activity}

The AgNps synthesised from ethanol extraction of rose (Rosa Indica) petals have potent anti-inflammatory activity by attenuating the production of superoxide anion $\left(\mathrm{O}^{-}\right)$and nitric oxide (NO) which was studied in rat peritoneal macrophages (Ramar et al., 2015). 
Table 2: Anticancer activity of AgNps synthesized from different sources.

\begin{tabular}{|c|c|c|c|c|}
\hline BIOLOGICAL SOURCE/METHOD & $\begin{array}{l}\text { SIZE } \\
\text { (NM) }\end{array}$ & SHAPE & CELL TYPE & REFERENCES \\
\hline $\begin{array}{l}\text { Extracts from Cucurbita maxima (petals), } \\
\text { Moringa oleifera (leaves), } \\
\text { Acorus calamus (rhizome). }\end{array}$ & $30-72$ & $\begin{array}{l}\text { Highly crystalline, roughly } \\
\text { spherical and cuboidal }\end{array}$ & $\begin{array}{l}\text { Epidermoid carcinoma cell } \\
\text { (A431) }\end{array}$ & $\begin{array}{l}\text { Nayak et al., } \\
2015\end{array}$ \\
\hline Caffeic acid (Plant Polyphenol)) & $3-10$ & Spherical & human hepatoma HepG2 & Guo et al., 2015 \\
\hline Aqueous extract of leaf of Andrographis echioides & -- & hexagonal & $\begin{array}{l}\text { Human breast adenocarcinoma } \\
\text { cancer cell line (MCF-7) }\end{array}$ & $\begin{array}{l}\text { Elangovan et al., } \\
2015\end{array}$ \\
\hline Ethanolic extraction of petals of Rosa Indica & $23.52-60.83$ & Crystalline and spherical & $\begin{array}{l}\text { human colon cancer cells (HCT } \\
15 \text { cells) }\end{array}$ & $\begin{array}{l}\text { Ramar et al., } \\
2015\end{array}$ \\
\hline Abelmoschus esculentus (L.) pulp extract & $3-11$ & Spherical & $\begin{array}{l}\text { Jurkat cell line (human T-cell } \\
\text { lymphoma) }\end{array}$ & $\begin{array}{l}\text { Mollick et al., } \\
2015\end{array}$ \\
\hline Citrate coated AgNps & 10 & -- & human lung cells (BEAS-2B) & Gliga et al., 2014 \\
\hline
\end{tabular}

Table 3: Antimicrobial activity of AgNps synthesized from different sources.

\begin{tabular}{|c|c|c|c|c|c|}
\hline BIOLOGICAL SOURCE/METHOD & $\begin{array}{l}\text { SIZE } \\
(\mathbf{N M})\end{array}$ & SHAPE & MICROBES NAME & $\begin{array}{l}\text { ZONE OF } \\
\text { INHIBITION } \\
\text { (MM) }\end{array}$ & REFERENCES \\
\hline $\begin{array}{l}\text { Extract from Cucurbita maxima (petals), } \\
\text { Moringa oleifera (leaves) } \\
\text { Acorus calamus (rhizome) }\end{array}$ & $30-72$ & $\begin{array}{l}\text { Highly crystalline, } \\
\text { roughly spherical } \\
\text { and cuboidal }\end{array}$ & $\begin{array}{l}\text { Bacillus subtilis, Escherichia coli, } \\
\text { Pseudomonas aeruginosa, Vibrio } \\
\text { cholera }\end{array}$ & -- & $\begin{array}{l}\text { Nayak et al., } \\
2015\end{array}$ \\
\hline $\begin{array}{l}\text { Aqueous extract of leaf of } \\
\text { Andrographis echioides }\end{array}$ & - & Hexagonal & $\begin{array}{l}\text { Escherichia coli, } \\
\text { Staphylococcus aureus } \\
\text { Salmonella typhi } \\
\text { Micrococcus luteus } \\
\text { Pseudomonas aeruginosa } \\
\end{array}$ & $\begin{array}{l}28, \\
23 \\
18 \\
15 \\
13\end{array}$ & $\begin{array}{l}\text { Elangovan et al., } \\
2015\end{array}$ \\
\hline $\begin{array}{l}\text { Ethanolic extraction of petals of } \\
\text { Rosa Indica }\end{array}$ & $\begin{array}{l}23.52- \\
60.83\end{array}$ & $\begin{array}{l}\text { Crystalline and } \\
\text { spherical }\end{array}$ & $\begin{array}{l}\text { Escherichia coli (MTCC-40) } \\
\text { Klebsiella pneumonia (MTCC-740) } \\
\text { Streptococcus mutans (MTCC-896) } \\
\text { Enterococcus faecalis (MTCC-439) }\end{array}$ & -- & $\begin{array}{l}\text { Ramar et al., } \\
2015\end{array}$ \\
\hline $\begin{array}{l}\text { Abelmoschus esculentus (L.) } \\
\text { pulp extract }\end{array}$ & $3-11$ & Spherical & $\begin{array}{l}\text { Bacillus subtilis (MTCC 736) } \\
\text { Bacillus cereus (MTCC 306) } \\
\text { Pseudomonas aeruginosa (MTCC 8158) } \\
\text { Micrococcus luteus (MTCC 1538) } \\
\text { Escherichia coli (MTCC 68) }\end{array}$ & $\begin{array}{l}33 \\
28 \\
26 \\
40 \\
19\end{array}$ & $\begin{array}{l}\text { Mollick et al., } \\
2015\end{array}$ \\
\hline $\begin{array}{l}\text { Aqueous root extract of } \\
\text { Delphinium denudatum }\end{array}$ & 85 & Spherical & $\begin{array}{l}\text { Bacillus cereus NCIM } 2106 \\
\text { Escherichia coli ATCC } 8739\end{array}$ & $\begin{array}{l}10 \\
08 \\
\end{array}$ & $\begin{array}{l}\text { Suresh } \text { et al., } \\
2014\end{array}$ \\
\hline $\begin{array}{l}\text { leaf extract of } \\
\text { Ocimum tenuiflorum }\end{array}$ & $25-40$ & Spherical & $\begin{array}{l}\text { Escherichia coli } \\
\text { Corynebacterium } \\
\text { Bacillus substilus } \\
\end{array}$ & $\begin{array}{l}10,15,20 \\
14,16,20 \\
19,24,26 \\
\end{array}$ & Patil et al., 2012 \\
\hline $\begin{array}{l}\text { Seeds of Elaeocarpus ganitrus } \\
\text { (Rudraksha) and } \\
\text { Foliage of Prosopis spicigera (Shami) }\end{array}$ & -- & -- & $\begin{array}{l}\text { Pseudomonas aeruginosa } \\
\text { Staphylococcus aureus }\end{array}$ & $\begin{array}{l}25,25 \\
21,21\end{array}$ & $\begin{array}{l}\text { Dwivedi } \text { et al., } \\
2013\end{array}$ \\
\hline
\end{tabular}

Table 4: Anticancer activity of gold nanparticles synthesized from different sources.

\begin{tabular}{|c|c|c|c|c|}
\hline BIOLOGICAL SOURCE/METHOD & $\begin{array}{l}\text { SIZE } \\
\text { (NM) }\end{array}$ & SHAPE & CELL TYPE & REFERENCES \\
\hline Areca catechu nut & 22.2 & Spherical & He La cell lines & Rajan et al., 2015 \\
\hline Kaempferol mediated & 16.5 & Spherical & MCF-7 (breat cancer cells) & Raghavan et al., 2015 \\
\hline $\begin{array}{l}\text { Gold nanopraticles modified with alpha-tocopheryl and arginine- } \\
\text { glycine-aspartic acid }\end{array}$ & 4 & Spherical & $\begin{array}{l}\text { U8MG - human primary } \\
\text { glioblastoma cell line }\end{array}$ & Zhu et al., 2015 \\
\hline
\end{tabular}

\section{GNP}

\section{Anticancer Study}

The biosynthesized gold nanopraticles (GNps) showed anticancer activity against He La cell lines and caused $66 \%$ of cell death at $100 \mu \mathrm{L}$ concentration. (Rajan et al., 2015). The anticancer activity of gold nanoparticles synthesized from different plant sources were represented in table 4. The GNps synthesised using a flavanol source, Kaempferol (3, 5, 7, 4 tetrahydroxyflavone) have potential anticancer activity against MCF-7 by inducing apoptosis by increasing in sub-G1 (hypodiploid) population. By inducing angiogenesis process they also inhibited vascular endothelial growth factor (VEGF) which was confirmed through chorioallantoic membrane assay (CAM). It also reduces the viability of cells of MCF-7 based on the dose and time depended manner (Raghavan et al., 2015). Multifunctional dendrimer-entrapped GNps (Au DENPs) modified with alphatocopheryl succinate ( $\alpha$-TOS) and arginine-glycine-aspartic acid (RGD) peptide have potential targeting towards the cancer cells which over express the $\alpha_{v} \beta_{3}$ integrin and inhibits the proliferation of cancer cell (U8MG - human primary glioblastoma cell line) by inducing apoptosis (Zhu et al., 2015). 


\section{Antimicrobial Study}

The biosynthesized GNps showed antibacterial activity against E. coli, K. Pneumonia, P. aeruginosa, Enterobacter sp. and S.aureus with respect to ZoI of $10,11,12,10$ and $14 \mathrm{~mm}$ respectively (Rajan et al., 2015). The antimicrobial activity of gold nanoparticles synthesized from a plant is given in the table 5 .

\section{Antioxidant study}

The GNps have showed potent free radical scavenging activity against the nitric oxide (NO) and 2,2-diphenyl-1picrylhydrazyl (DPPH) (Rajan et al., 2015).

\section{Catalytic Activity}

The GNps showed an excellent catalytic activity (reduction activity) of organic dye such as MB, MO, EY and 4-NP and this can be used in removing hazardous environmental pollutants (Rajan et al., 2015).

\section{Larvicidal Activity}

GNps synthesised from Cymbopogan citratus have excellent antiparasitic activity against the $C$. quinquefasciatus (filaraial vector) ( $\mathrm{LC}_{50}$ and $\mathrm{LC}_{90}: 1.08$ and $2.76 \mathrm{ppm}$ ) while leaf extract of Anthocephalus cadamba exhibited 21.82 and 79.52ppm of $\mathrm{LC}_{50}$ and $\mathrm{LC}_{90}$ against third instar larvae of $C$. quinquefasciatus (Naresh Kumar et al., 2012). The larvicidal activity of gold nanoparticles synthesized from a plant is given in the table 6 .

\section{IRON NANOPARTICLES (INPs)}

\section{Anticancer Study}

The in vitro cytotoxicity of non-toxic $\beta$-cyclodextrin assemblied magnetic $\mathrm{Fe}_{3} \mathrm{O}_{4}$ nanoparticles ( $\beta$-CD-MNPs) had shown greater anticancer activity against MCF-7 and it was also used as drug delivery system in Cancer therapy (Wang et al., 2015). The anticancer activity of iron nanoparticles synthesized from different plant is tabulated in table 7. A nanocomposites synthesized through layer-by-layer technique called multifunctional peptide-fluorescent-magnetic nanocomposites ( $\mathrm{Fe}_{3} \mathrm{O}_{4} @$ PEI@Cy5.5@PEG@HCBP-1 NPs, HPCPMNPs) have strong influence on the the cell viability of human embryonic lung fibroblast cell line (HLF) and human mesenchymal stem cell (MSC) at the concentration of $0.5 \mathrm{mg} / \mathrm{mL}$ and $1 \mathrm{mg} / \mathrm{mL}(\mathrm{p}<0.01)$ treatment. HPCPMNPs also have the capability of targeting or detecting the human lung cancer cell line (H460) of about $80 \%$. This indicates that these nanocomoposites can be used in cancer diagnostic agent by modifying diverse fluorescence dyes and targeting ligands on its surface (Zhou et al., 2015). Semkina et al. (2015) reported the anticancer activity of Doxorubin (Doxanticancer drug) loaded superparamagnetic iron oxide $\left(\mathrm{Fe}_{3} \mathrm{O}_{4}\right)$ magnetic nanoparticles (MNPs) with Bovine Serum Albumin (BSA) and Polyethylene Glycol (PEG) called MNP-BSA@DoxPEG against Dox-resistance (C6 - rat glial tumour cells induced by N-nitrosomethylurea) and Dox-sensitive (HEK293-human embryonic kidney cells) by inhibiting the cell viability.

\section{OTHER NANOPARTICLES}

\section{Anticancer Study}

The anticancer activity of nickle doped cerium oxide $\left(\mathrm{Ni}_{\mathrm{x}}\right.$ $\left.\mathrm{Ce}_{1{ }^{-} \mathrm{x}} \mathrm{O} 2\right)$ had shown that there was $55 \%$ reduction of neuroblastoma cancer cell line viability without exhibiting harm to the normal healthy human embryonic kidney 293 cell-HEK293) (Abbas et al., 2015).

PST-Dox (Galactoxyloglucan, PST001-conjugated Dox) nanoparticle showed a potential anticancer activity against lung adinocarcinoma (A549) and metastatic melanoma (A375) by inducing apoptosis. A PST-Dox nanoparticle was reported with non-toxic to normal lymphocytes up to certain dosage (Joseph et al., 2015).

DPM@PL (DTX loaded methoxy polyethylene glycol-ss-vitamin E succinate (PSV) micelles (DPM) @ PPV-based liposomes) have shown inhibition of in situ tumour growth and pulmonary metastasis formation by DTX- inducing the apoptosis and decreased level of metastasis-promoting protein formation. They also have $81 \%$ and $92 \%$ inhibition rate against the tumour volume and lung metastasis. It doesn't show any systemic toxicity. This indicates that the DPM@PL nanoparticle which can be used as a delivery systems for breast cancer therapy (Xu et al., 2015).

Silica nanopraticles (Dual-SiNPs) which was synthesised using dual aptamer modifier have excellent sensitive detection against two breast cancer cell such as mucin 1 (MUC1) (+) and human epidermal growth factor receptor $2(\mathrm{HER} 2)(+)$ cell lines at the concentration of 1 cell $/ 100 \mu \mathrm{L}$. This indicates its role in diagnosis and prognosis of breast cancer (Jo et al., 2015).

In vitro study with DTX-loaded Gal-pD-TPGS-PLA/NPs (galactosamine conjugated polydopamine-modified NPs synthesized using D- $\alpha$-tocopherol polyethylene glycol 1000 succinate-poly(lactide) showed potent anticancer activity against HepG2 on heptoma-bearing nude mice by decreasing the tumour size. It also had the higher efficiency of uptaking liver cancer cell line HepG2 which was confirmed by flow cytometry and confocal laser scanning microscopy. It reveals its drug delivery system in liver cancer (Zhu et al., 2015).

By nab technology, human serum albumin loaded with lapatinib (Tykerb - tyrosine kinase inhibitor of HER2 and EFGR) was developed called LHNPs which has excellent anti-tumour efficiency by ceasing the proliferation of HER 2 by over expressing $\mathrm{SKBr} 3$ (human breast cancer cell line) in tumour-bearing mice. They also suggested that it doesn't have hepatic or kidney toxicity (Wan et al., 2015). LFC/CMC NPs (LFC131 peptide surface conjugated O-carboxymethyl chitosan nanoparticles (O-CMC NP)] have capability of increasing cancer cell death through selective delivery of DTX.

It also has higher efficiency of uptaking tumour cell by receptor mediated interaction of LFC131 with the CXCR4 which is over expressed in cancer cell and by inducing caspase- 3 activity in A549 cancer cells. They have also suggested that the NPs have greater cell apoptosis by inducing apoptotic and necrotic cell death (Wang et al., 2015). 
Table 5: Antimicrobial activity of gold nanoparticles synthesized from different sources.

\begin{tabular}{|c|c|c|c|c|c|}
\hline $\begin{array}{c}\text { BIOLOGICAL } \\
\text { SOURCE/METHOD }\end{array}$ & $\begin{array}{l}\text { SIZE } \\
(\mathbf{N M})\end{array}$ & SHAPE & MICROBES NAME & ZONE OF INHIBITION (MM) & REFERENCES \\
\hline \multirow{5}{*}{ Areca catechu nut } & \multirow{5}{*}{22.2} & \multirow{5}{*}{ Spherical } & Escherichia coli & 10 & \multirow{5}{*}{ Rajan et al., 2015} \\
\hline & & & Staphylococcus aureus & 14 & \\
\hline & & & Enterobacter & 10 & \\
\hline & & & Pseudomonas aeruginosa & 12 & \\
\hline & & & K.pneumonia & 11 & \\
\hline
\end{tabular}

Table 6: Larvicidal activity of gold nanoparticles synthesized from different sources.

\begin{tabular}{|c|c|c|c|c|c|}
\hline \multirow{2}{*}{$\begin{array}{l}\text { BIOLOGICAL } \\
\text { SOURCE/METHOD }\end{array}$} & \multirow{2}{*}{$\begin{array}{l}\text { SIZE } \\
\text { (NM) }\end{array}$} & \multirow{2}{*}{$\begin{array}{l}\text { NAME OF MOSQUITO } \\
\text { SPECIES }\end{array}$} & \multicolumn{2}{|c|}{$\begin{array}{l}\text { LETHAL CONCENTRATION (LC) } \\
\text { (MG/L) / PPM }\end{array}$} & \multirow[t]{2}{*}{ REFERENCES } \\
\hline & & & $\mathrm{LC}_{50}$ & $\mathrm{LC}_{90}$ & \\
\hline Leaf extract of Cymbopogan citratus & & Culex quinquefasciatus & 1.08 & 2.76 & Naresh kumar et al., 2012 \\
\hline
\end{tabular}

Table 7: Anticancer activity of Iron Nanoparticles.

\begin{tabular}{cccc}
\hline BIOLOGICAL SOURCE/METHOD & $\begin{array}{c}\text { SIZE } \\
\text { (NM) }\end{array}$ & SHAPE & CELL TYPE \\
\hline Non-toxic $\beta$-cyclodextrin assemblied magnetic $\mathrm{Fe}_{3} \mathrm{O}_{4}$ nanoparticles & 12 & Spherical & $\begin{array}{c}\text { Michigan Cancer Foudation-7 } \\
\text { (MCF-7- a breast cancer cell line) }\end{array} \quad$ Wang et al., 2015 \\
\hline $\begin{array}{c}\text { Multifunctional peptide-fluorescent-magnetic nanocompositesusing } \\
\text { layer by layer method }\end{array}$ & 22.3 & $\begin{array}{c}\text { Human embryonic lung fibroblast } \\
\text { cell line (HLF), human } \\
\text { mesenchymal stem cell (MSC) } \\
\text { and human lung cancer cell line } \\
\text { (H460) }\end{array}$ \\
\hline $\begin{array}{c}\text { Zhou } \text { et al., 2015 } \\
\text { Doxorubin loaded Superparamagnetic iron oxide magnetic } \\
\text { nanoparticles with Bovine Serum Albumin and Polyethylene Glycol }\end{array}$ & 85 & - & $\begin{array}{c}\text { Rat glial tumour cells and } \\
\text { HEK293-human embryonic } \\
\text { kidney cells }\end{array}$ \\
\hline
\end{tabular}

Table 8: Anticancer and larvicidal activity of nanoparticles of different origin.

\begin{tabular}{|c|c|c|c|c|}
\hline $\begin{array}{c}\text { BIOLOGICAL } \\
\text { SOURCE/METHOD } \\
\end{array}$ & $\begin{array}{l}\text { SIZE } \\
\text { (NM) }\end{array}$ & SHAPE & CELL TYPE & REFERENCES \\
\hline $\begin{array}{l}\text { Nickle doped cerium oxide using } \\
\text { Co-precipitation method }\end{array}$ & 22 & Spherical & H-SY-5Y neuroblastoma cancer cell line & Abbas et al., 2015 \\
\hline $\begin{array}{c}\text { Galactoxyloglucan, PST001- } \\
\text { conjugated Dox }\end{array}$ & 10 & Spherical & $\begin{array}{l}\text { Lung adinocarcinoma (A549) and metastatic melanoma } \\
\text { (A375) }\end{array}$ & Joseph et al., 2015 \\
\hline $\begin{array}{c}\text { DTX loaded methoxy } \\
\text { polyethylene glycol-s-s-vitamin } \\
\text { E succinate (PSV) micelles } \\
\text { (DPM) @ PPV-based liposomes }\end{array}$ & 113.3 & Shrapnel & in situ tumour growth and pulmonary metastasis & Xu et al., 2015 \\
\hline $\begin{array}{l}\text { Gold Nanoparticle synthesised } \\
\text { using Dual aptamer modifier }\end{array}$ & 70 & -- & $\begin{array}{l}\text { breast cancer cell such as mucin } 1(\text { MUC1) }(+) \text { and human } \\
\text { epidermal growth factor receptor } 2(\mathrm{HER} 2)(+) \text { cell lines }\end{array}$ & Jo et al., 2015 \\
\hline $\begin{array}{l}\text { galactosamine conjugated } \\
\text { polydopamine-modified NPs } \\
\text { synthesized using D- } \alpha- \\
\text { tocopherol polyethylene glycol } \\
1000 \text { succinate-poly(lactide) }\end{array}$ & 209.4 & Spherical & HepG2 - heptocellular carcinoma cell & Zhu et al., 2015 \\
\hline $\begin{array}{l}\text { Human serum albumin loaded } \\
\text { with lapatinib }\end{array}$ & $\begin{array}{c}145.2 \pm \\
4.55\end{array}$ & -- & human breast cancer cell line - HER2 & Wan et al., 2015 \\
\hline $\begin{array}{l}\text { LFC131 peptide surface } \\
\text { conjugated O-carboxymethyl } \\
\text { chitosan }\end{array}$ & $189 \pm 3.6$ & Spherical & A549 lung cancer cells & Wang et al., 2015 \\
\hline $\begin{array}{l}\text { Cobalt acetate solution and } \\
\text { Bacillus thuringiensis }\end{array}$ & 85.3 & $\begin{array}{l}\mathrm{LC}_{50}: 29.16,8.12,3.59 \\
\mathrm{LC}_{50}: 34.61,6.94,2.87\end{array}$ & $\begin{array}{c}\text { Anopheles subpictus } \\
\text { Aedes aegypti }\end{array}$ & $\begin{array}{l}\text { Marimuthu et al., } \\
2013\end{array}$ \\
\hline
\end{tabular}

\section{Larvicidal activity}

Cobalt nanoparticles (CoNps) synthesised using cobalt acetate solution and $B$. thuringiensis (bio control agent) have potential larvicidal activity against fourth instar larvae of $A$. subpictus (malaria vector) and $A$. aegypti (dengue vector) $\left(\mathrm{LC}_{50}\right.$ values of $29.16,8.12,3.59 \mathrm{mg} / \mathrm{L} ; 34.61,6.94$, and $2.87 \mathrm{mg} / \mathrm{L}$ ) (Marimuthu et al., 2013). The anticancer and larvicidal activity of other nanoparticles synthesized in different sources is represented in the table 8 .

\section{CONCLUSION}

In this review, a summary of various nanoparticles synthesised from different different biological and non biological 
sources. Based on the bioactivity, they were listed under different categories such as larvicidal agents, anti-cancer, anti-bacterial, anti-inflammatory, antioxidant and catalytic activity which were discussed in detail. In search of novel biomolecules for the welfare of human beings, active metal of nanosized materials of different sources have been extensively implemented and studied for biological activities against wide range of pathogenic agents and carriers/vectors. This review addressed the prosperous role of nanoparticles in different health sectors of human beings such as vector borne disease, cancer and infectious diseases. As anticipated by Benelli (2016), wide range of research has been executed by the researchers for the benefit of human beings and was observed in this complete review on nanoparticles. Hence nanoparticles playing a dynamic role in treatment and diagnostic of various threatening diseases and soon will occupy an inevitable role in healthcare development than now.

\section{ACKNOWLEDGEMENT}

The authors express their sincere gratitude to Dr. A. Vijaya Anand, Head Department of Human Genetics and Molecular Biology and Bharathiar University for giving moral support in structuring the review.

\section{Financial support and sponsorship: Nil.}

Conflict of Interests: There are no conflicts of interest.

\section{REFERENCE}

Suganya G, Karthi S, Shivakumar MS. Larvicidal potential of AgNps synthesized from Leucas aspera leaf extracts against dengue vector Aedes aegypti. Parasitol Res. 2014; 113:1673-1679

Suresh U, Murugan K, Benelli G, Nicoletti M, Barnard DR, Panneerselvam C. Tackling the growing threat of dengue: Phyllanthus niruri-mediated synthesis of AgNps and their mosquitocidal properties against the dengue vector Aedes aegypti (Diptera: Culicidae). Parasitol Res. 2015; 114:1551-1562

Veerakumar K, Govindarajan M, Rajeswary M. Green synthesis of AgNps using Sida acuta (Malvaceae) leaf extract against Culex quinquefasciatus, Anopheles stephensi, and Aedes aegypti (Diptera: Culicidae). Parasitol Res. 2013; 112:4073-4085

Gnanadesigan M, Anand M, Ravikumar S, Maruthupandy M, Vijayakumar V, Selvam S. Biosynthesis of AgNps by using mangrove plant extract and their potential mosquito larvicidal property. Asian Pacific Journal of Tropical Medicine. 2011; 799-803.

Abbas F, Jan T, Iqbal J, Ahmad I, Sajjad M, Naqvi H. Facile synthesis of ferromagnetic $\mathrm{Ni}$ doped $\mathrm{CeO}_{2}$ nanoparticles with enhanced anticancer activity. Applied Surface Science. 2015; 357: 931-936

Benelli G, Iacono AL, Canale A, Mehlhorn H. Mosquito vectors and the spread of cancer: an overlooked connection? Parasitol Res. 2016;115:2131-2137

Joseph MM, Aravind SR, George SK, Pillai KR, Mini S, Sreelekha TT. Anticancer activity of galactoxyloglucan polysaccharideconjugated doxorubicin nanoparticles: Mechanistic insights and interactome analysis. European Journal of Pharmaceutics and Biopharmaceutics. 2015; 93: 183-195

Santhoshkumar T, Rahuman AA, Bagavan A, Marimuthu S, Jayaseelan C, Kirthi AV. Evaluation of stem aqueous extract and synthesized AgNps using Cissus quadrangularis against Hippobosca maculata and Rhipicephalus (Boophilus) microplus. Experimental Parasitology. 2012; 132: 156-165
Roopan SM, Rohit, Madhumitha G, Rahuman AA, Kamaraj C, Bharathi A. Low-cost and eco-friendly phyto-synthesis of AgNps using Cocos nucifera coir extract and its larvicidal activity. Industrial Crops and Products. 2012; 43: 631-635

Velayutham K, Rahuman AA, Rajakumar G, Roopan SM, Elango G, Kamaraj C. Larvicidal activity of green synthesized AgNps using bark aqueous extract of Ficus racemosa against Culex quinquefasciatus and Culex gelidus. Asian Pacific Journal of Tropical Medicine. 2012; 95-101

Suman TY, Elumalai D, Kaleena PK, Rajasree SRR. GC-MS analysis of bioactive components and synthesis of AgNps using Ammannia baccifera aerial extract and its larvicidal activity against malaria and filariasis vectors. Industrial Crops and Products. 2013; 47: 239 $-245$

Suresh G, Gunasekar PH, Kokila D, Prabhu D, Dinesh D, Ravichandran N. Green synthesis of AgNps using Delphinium denudatum root extract exhibits antibacterial and mosquito larvicidal activities. Spectrochimica Acta Part A: Molecular and Biomolecular Spectroscopy. 2014; 127:61-66

Vimala R.T.V., Sathishkumar G, Sivaramakrishnan S. Optimization of reaction conditions to fabricate nano-silver using Couroupita guianensis Aubl. (leaf \& fruit) and its enhanced larvicidal effect. Spectrochimica Acta Part A: Molecular and Biomolecular Spectroscopy. 2014; 135:110-115

Veerakumar K and Govindarajan M. Adulticidal properties of synthesized AgNps using leaf extracts of Feronia elephantum (Rutaceae) against filariasis, malaria, and dengue vector mosquitoes. Parasitol Res. 2014; 113:4085-4096

Dinesh D, Murugan K, Madhiyazhagan P, Panneerselvam C, Kumar PM, Nicoletti M. Mosquitocidal and antibacterial activity of greensynthesized AgNps from Aloe vera extracts: towards an effective tool against the malaria vector Anopheles stephensi? Parasitol Res. 2015; 114:1519-1529

Santhosh SB, Yuvarajan R, Natarajan D. Annona muricata leaf extract-mediated AgNps synthesis and its larvicidal potential against dengue, malaria and filariasis vector. Parasitol Res. 2015. DOI 10.1007/s00436-015-4511-2

Priyadarshini KA, Murugan K, Panneerselvam C, Ponarulselvam S, Hwang JS, Nicoletti M. Biolarvicidal and pupicidal potential of AgNps synthesized using Euphorbia hirta against Anopheles stephensi Liston (Diptera: Culicidae). Parasitol Res. 2012; 111: 997-1006

Kumar RK, Nattuthurai N, Gopinath P, Mariappan T. Synthesis of eco-friendly AgNps from Morinda tinctoria leaf extract and its larvicidal activity against Culex quinquefasciatus. Parasitol Res. 2014; 114: 411-417

Muthukumaran U, Govindarajan M, Rajeswary M. Mosquito larvicidal potential of AgNps synthesized using Chomelia asiatica (Rubiaceae) against Anopheles stephensi, Aedes aegypti, and Culex quinquefasciatus (Diptera: Culicidae). Parasitol Res. 2014; 114: 989-999

Veerakumar K, Govindarajan M, Rajeswary M, Muthukumaran U. Low-cost and eco-friendly green synthesis of AgNps using Feronia elephantum (Rutaceae) against Culex quinquefasciatus, Anopheles stephensi, and Aedes aegypti (Diptera: Culicidae). Parasitol Res. 2014; 113: $1775-1785$

Muthukumaran U, Govindarajan M, Rajeswary M, Hoti SL. Synthesis and characterization of AgNps using Gmelina asiatica leaf extract against filariasis, dengue, and malaria vector mosquitoes. Parasitol Res. 2015; 114: 1817-1827

Haldar KM, Haldar B, Chandra G. Fabrication, characterization andmosquito larvicidal bioassay of AgNps synthesized from aqueous fruit extract of putranjiva, Drypetes roxburghii (Wall.). Parasitol Res. 2013; 112: $1451-1459$

Santhoshkumar T, Rahuman AA, Rajakumar G, Marimuthu S, Bagavan A, Jayaseelan C. Synthesis of AgNps using Nelumbo nucifera leaf extract and its larvicidal activity against malaria and filariasis vectors. Parasitol Res. 2010; 108: 693-702

Chitra G, Balasubramani G, Ramkuma R, Sowmiya R, Perumal P. Mukia maderaspatana (Cucurbitaceae) extract-mediated synthesis of 
AgNps to control Culex quinquefasciatus and Aedes aegypti (Diptera: Culicidae). Parasitol Res. 2015; 114: 1407-1415

Suganya A, Murugan K, Kovendan K, Kumar PM, Hwang JS. Green synthesis of AgNps using Murraya koenigii leaf extract against Anopheles stephensi and Aedes aegypti. Parasitol Res. 2012; 112: 13851397

Roni M, Murugan K, Panneerselvam C, Subramaniam J, Hwang JS. Evaluation of leaf aqueous extract and synthesized AgNps using Nerium oleander against Anopheles stephensi (Diptera: Culicidae). Parasitol Res. 2012; 112: 981-990

Nayak D, Pradhan S, Ashe S, Rauta PR, Nayak B. Biologically synthesised $\mathrm{AgNps}$ from three diverse family of plant extracts and their anticancer activity against epidermoid A431 carcinoma. Journal of Colloid and Interface Science. 2015; 457: 329-338

Guo D, Dou D, Ge L, Huang Z, Wang L, Gu N. A caffeic acid mediated facile synthesis of AgNps with powerful anti-cancer activity. Colloids and Surfaces B: Biointerfaces. 2015; 134: 229-234

Elangovan K, Elumalai D, Anupriya S, Shenbhagaraman R, Kaleena PK, Murugesan K. Phyto mediated biogenic synthesis of AgNps using leaf extract of Andrographis and its bio-efficacy on anticancer and antibacterial activities. Journal of Photochemistry and Photobiology B: Biology. 2015; 151: 118-124

Ramar M, Manikandan B, Raman T, Arunagirinathan K, Prabhu NM, Basu MJ. Biosynthesis of AgNps using ethanolic petals extract of Rosa Indica and characterization of its antibacterial, anticancer and antiinflammatory activities. Spectrochimica Acta Part A: Molecular and Biomolecular Spectroscopy. 2015; 138: 120-129

Gliga AR, Skoglund S, Wallinder IO, Fadeel B, Karlsson HL. Size-dependent cytotoxicity of AgNps in human lung cells: the role of cellular uptake, agglomeration and $\mathrm{Ag}$ release. Particle and Fibre Toxicology. 2014; 11(11): 1-17

Mollick MR, Rana D, Dash SK, Chattopadhyay S, Bhowmick B, Maity D. Studies on green synthesized AgNps using Abelmoschus esculentus (L.) pulp extract having anticancer (in vitro) and antimicrobial applications. Arabian Journal of Chemistry. 2015. http://dx.doi.org/10.1016/j.arabjc.2015.04.033

Borah D, Deka P, Bhattacharjee P, Changmai A, Yadav RNS. Ocimum sanctum mediated silver nano particles showed better antimicrobial activities compared to citrate stabilized silver nano particles against multidrug resistant bacteria. Journal of Pharmacy research. 2013; 7: 478-482

Patil RS, Kokate MR, Kolekar SS. Bioinspired synthesis of highly stabilized AgNps using Ocimum tenuiflorum leaf extract and their antibacterial activity. Spectrochimica Acta Part A. 2012; 91: 234-238

Dwivedi P, Narvia SS, Tewari RP. Phytofabrication characterization and comparative analysis of $\mathrm{Ag}$ nanoparticles by diverse biochemicals from Elaeocarpus ganitrus Roxb., Terminalia arjuna Roxb., Pseudotsuga menzietii, Prosopis spicigera, Ficus religiosa, Ocimum sanctum, Curcuma longa. Industrial Crops and Products. 2013; 54: 22-31

Rajan A, Vilas V, Philip D. Studies on catalytic, antioxidant, antibacterial and anticancer activities of biogenic gold nanoparticles. Journal of Molecular Liquids. 2015; 212: 331-339

Raghavan BS, Kondath S, Anantanarayanan R, Rajaram R. Kaempferol mediated synthesis of gold nanoparticles and their cytotoxic effects on MCF-7 cancer cell line. Process Biochemistry. 2015. http://dx.doi.org/10.1016/j.procbio.2015.08.003
Zhua J, Fub F, Xiongb Z, Shenb M, Shi X. Dendrimerentrapped gold nanoparticles modified with RGD peptide and alphatocopheryl succinate enable targeted theranostics of cancer cells. Colloids and Surfaces B: Biointerfaces. 2015; 133: 36-42

Kumar AN, Jeyalalitha T, Murugan K, Madhiyazhagan P. Bioefficacy of plant-mediated gold nanoparticles and Anthocepholus cadamba on filarial vector, Culex quinquefasciatus (Insecta: Diptera: Culicidae). Parasitol Res. 2012; 112: 1053-1063

Wang C, Huang L, Song S, Saif B, Zhou Y, Dong C. Targeted delivery and $\mathrm{pH}$-responsive release of stereoisomeric anti-cancer drugs using $\beta$-cyclodextrin assemblied $\mathrm{Fe}_{3} \mathrm{O}_{4}$. Appl. Surf. Sci. 2015. http://dx.doi.org/10.1016/j.apsusc.2015.09.189

Zhou Z, Chen L, Wang A, Maa Y, Zhang H, Zhu Y. Multifunctional fluorescent magnetic nanoparticles for lung cancer stem cell research. Colloids and Surfaces B: Biointerfaces. 2015; 134: 431-439

Semkina A, Abakumov M, Grinenko N, Abakumov A, Skorikov A, Mironova E. Core-shell-corona doxorubicin-loaded superparamagnetic $\mathrm{Fe}_{3} \mathrm{O}_{4}$ nanoparticles for cancer theranostics. Colloids and Surfaces B: Biointerfaces. 2015; 136: 1073-1080

Xu P, Meng Q, Sun H, Yin Q, Yu H, Zhang Z. Shrapnel nanoparticles loading docetaxel inhibit metastasis and growth of breast cancer. Biomaterials. 2015; 64: 10-20

Jo H, Her J, Ban C. Dual aptamer-functionalized silica nanoparticles for the highly sensitive detection of breast cancer. Biosensors and Bioelectronics. 2015; 71: 129-136

Zhu H, Tao W, Zhang H, Liu G, Wang T, Zhang L. Docetaxel (DTX)-loaded polydopamine-modified TPGS-PLA nanoparticles as a targeted drug delivery system for the treatment of liver cancer. Acta Biomaterialia. 2015. http://dx.doi.org/10.1016/j.actbio.2015.11.031

Wan X, Zheng X, Pang X, Zhang Z, Zhang Q. Incorporation of lapatinib into human serum albumin nanoparticles with enhanced antitumor effects in HER2-positive breast cancer. Colloids and Surfaces B: Biointerfaces. 2015; 136: 817-827

Wang RT, Zhi XY, Yao SY, Zhang Y. LFC131 peptideconjugated polymeric nanoparticles for the effective delivery of docetaxel in CXCR4 overexpressed lung cancer cells. Colloids and Surfaces B: Biointerfaces. 2015; 133: 43-50

Marimuthu S, Rahuman AA, Kirthi AV, Santhoshkumar T, Jayaseelan C, Rajakumar G. Eco-friendly microbial route to synthesize cobalt nanoparticles using Bacillus thuringiensis against malaria and dengue vectors. Parasitol Res. 2013; 112(12): 4105-12.

How to cite this article:

Vignesh M and Moorthi PV. An Overview of Naturally Synthesized Metallic Nanoparticles. J App Pharm Sci, 2017; 7 (06): 229-237. 